\title{
Abdominal Massage Alleviates Skeletal Muscle Insulin Resistance by Regulating the AMPK/SIRT1/PGC-1a Signaling Pathway
}

\author{
Yiran Han $\mathbb{D}^{1} \cdot$ Zeyuan Lu $\mathbb{1}^{2} \cdot$ Shaotao Chen $\mathbb{D}^{1} \cdot$ Chongwen Zhong $\mathbb{D}^{1} \cdot$ Minghui Yan $\mathbb{D}^{1} \cdot$ Heran Wang $\mathbb{D}^{1} \cdot$ \\ Meng Meng $\mathbb{1}^{1} \cdot$ Mingjun Liu $\mathbb{1}^{1}$
}

Received: 23 November 2020 / Accepted: 31 March 2021 / Published online: 8 May 2021

(c) The Author(s) 2021

\begin{abstract}
Abdominal massage (AM), a traditional Chinese medicine-based treatment method, has received considerable attention in the recent years. The aim of the present study was to investigate the effect of AM on high-fat diet (HFD)-induced insulin resistance (IR) in comparison with resveratrol (RSV) treatment. Forty-eight male Sprague-Dawley rats were randomly divided into the following four groups: standard chow diet (control group), high-fat diet (model group), HFD + abdominal massage (AM group), and HFD + resveratrol (RSV group). A rat model of IR was established by feeding HFD to rats for 8 weeks followed by treatment with AM or RSV for 4 weeks. The underlying HFD-induced IR molecular mechanisms were studied in rat serum and skeletal muscles. RSV and AM significantly improved glucose intolerance, hyperglycemia, obesity, and significantly reduced lipid accumulation [triglyceride (TC), total cholesterol (TG), low-density lipoprotein cholesterol (LDL-C), and high-density lipoprotein cholesterol (HDL-C)], adipocytokine [free fatty acids (FFA), adiponectin (ADPN)] and serum pro-inflammatory cytokines (IL-6 and TNF- $\alpha$ ) secretion. In addition, AM activated the AMPK/SIRT1 signaling pathway in rat skeletal muscle. In conclusion, our results showed that AM could improve IR by regulating the secretion of adipocytokines, pro-inflammatory cytokines as well as related signaling pathways in the skeletal muscle of rats, which might provide insights into development of new treatment methods for the clinical treatment of IR.
\end{abstract}

Keywords Abdominal massage $\cdot$ Insulin resistance $\cdot$ Inflammatory cytokines $\cdot$ Adipocytokine $\cdot$ AMPK/SIRT1/PGC- $1 \alpha$

\section{Introduction}

Insulin resistance (IR) is a complex metabolic disorder associated with obesity, nonalcoholic fatty liver disease, atherosclerosis, and type 2 diabetes mellitus (T2DM) [1]. Although the precise mechanism of IR remains unclear, the adenosine monophosphate-activated protein kinase/Sirtuin1 (AMPK/SIRT1) pathway is known to play a pivotal role in IR [2-6]. In recent years, increasing evidence has suggested that inflammatory responses, especially those mediated by adipocytokines, play a key role in the development of IR

Mingjun Liu

liumingjun123@hotmail.com

1 Departments of Acupuncture and Massage, Changchun University of Chinese Medicine, Changchun 130117 Jilin Province, PR China

2 Department of Pharmacology, School of Pharmaceutical Sciences, Jilin University, Changchun, Jilin Province 130021, PR China
[7, 8]. Adipocytokines are the cytokines released from adipose tissue, including leptin, adiponectin, acylation stimulating protein, and omentin along with tumor necrosis factor (TNF), interleukin-6 (IL-6), and interleukin-1 IL-1, which can block the activation of the AMPK/SIRT1 insulin signaling pathway and induce IR $[9,10]$.

Weight gain can lead to the development of morbid obesity, which in early stages can be managed through therapy, diet, physical exercise, and cognitive-behavioral therapy, without the need for medication, endoscopy, or surgery [11]. However, considering the difficulty of maintaining long-term calorierestricted diets, non-pharmacological interventions and lifestyle changes have received considerable attention for the management of weight gain and related disorders [12]. A substantial number of studies have indicated that massage therapy is beneficial in various conditions, such as metabolic disorders, pain syndromes, immune diseases, and aging-related health issues [13]. Moderate massage therapy has been shown to exert positive effects, including pain relief from various syndromes, increased concentration, decreased depression, and 
enhanced immune function (increased proportion of natural killer cells and natural killer cell activity) [14].

Traditional Chinese medicine massage therapy has been widely used in clinics as an external treatment [15-17]. Different techniques have been used in clinical treatment and in the prevention of several disease conditions with an aim to stimulate body meridian, improve blood circulation, boost metabolism, regulate the endocrine system, improve the function of autonomic nerves, and to eliminate the symptoms of systemic or local discomfort [18-20]. From the perspective of modern medicine, the abdomen is extremely rich in nerve cells and is also termed as "the second brain" [21]. Abdominal massage (AM) is defined as a non-invasive gentle massaging of abdomen performed to treat various conditions, such as obesity, T2DM, fatigue syndrome, and other medical problems [22-25]. AM can promote glucose and lipid metabolism and is shown to be effective in the clinical treatment of insulin-resistant obese patients [26, 27]. In our previous study, we found that AM significantly increased the expression of SIRT1 mRNA in skeletal muscle and triggered strong anti-inflammatory responses in Sprague-Dawley (SD) rats [28]. To the best of our knowledge, this is the first study to evaluate the effect of AM on IR. In the present study, we aimed to investigate the effects of AM on high-fat diet (HFD)-induced IR and the related mechanisms in comparison with resveratrol (RSV) intervention in SD rats.

\section{Materials and Methods}

\section{Reagents}

RSV (80051933) was purchased from Celex Laboratories Inc., and triglyceride (TC), total cholesterol (TG), lowdensity lipoprotein cholesterol (LDL-C), and high-density lipoprotein cholesterol (HDL-C) were purchased from Nanjing Jiancheng Bioengineering Institute, Nanjing, China (A110-A113); TNF- $\alpha$ (05619b), IL-6 (05621b), adiponectin (ADPN, 03586b), free fatty acids (FFA, 03247b), and GTX ELISA kits were purchased from Yaji Biological Technology Co., Ltd, Shanghai, China; PhosphoPlus ${ }^{\circledR}$ AMPK $\alpha$ (Thr172) antibody duet (8208) was purchased from Cell Signaling Technology, Inc., Shanghai, China; SIRT1 (13161-1-AP) and PGC-1 $\alpha$ (63369-1-LG) primary antibodies were purchased from Proteintech Group Inc, China; GAPDH primary antibody was purchased from Weikesaisi Technology Inc., Wuhan, China; radioimmunoprecipitation assay (RIPA) lysis buffer (WB-0071), horseradish peroxidase (HRP)-conjugated goat-anti rabbit/mouse secondary antibodies (AB-0041, AB-0021) were purchased from Dingguochangsheng Biotechnology Co., Ltd, Beijing, China; RNA extraction kit (R1051) was purchased from
Dongsheng Biotech Inc.; and the TransScript ${ }^{\circledR}$ Green TwoStep qRT-PCR SuperMix (AQ201-01) was purchased from Transgen Biotech Inc., Beijing, China.

\section{Animals and Models}

All animal experimental procedures were performed in accordance with the guidelines for care and use of laboratory animals of Changchun University of Traditional Chinese Medicine and approved by the Animal Experimental Ethics Committee (Approval No. 2020194). The rats (8week-old male SD rats, weighing 180-220 g) were raised by the College of Pharmacy, Jilin University, Changchun, China. Rats had free access to prescribed food and water under standard temperature $\left(24^{\circ} \mathrm{C}\right)$, relative humidity (50\%), and light (light-dark cycle) conditions. After subjection to adaptive feeding for 1 week, rats were randomly divided into a normal diet group (NF, $n=12)$ and a high-fat group (HDF, $n=36$ ); NF group rats were provided with normal feed (energy density: $3.8 \mathrm{kcal} / \mathrm{g}$; composition: $70 \%$ carbohydrate, $20 \%$ protein, and $10 \%$ fat); HDF group rats were provided with a HFD (energy density: $5.4 \mathrm{kcal} / \mathrm{g}$; composition: $38.5 \%$ carbohydrate, $15 \%$ protein, and $46.5 \%$ fat [29]). Diets were obtained from National Resource Center for Rodent Laboratory Animal. After subjection to 8 weeks of feeding, the glucose infusion rate was determined by performing glucose clamp assay in 36 rats from the HDF group and was found to be significantly lower than the NF group rats. These HDF group rats $(n=36)$ were then randomly divided into the model, RSV, and AM groups $(n=12)$. Twelve rats from the NF group were treated as controls. Animal experiments were conducted in accordance with laboratory animal care and user guidelines from the National Institutes of Health and were approved by the Ethics Committee of Changchun University of Chinese Medicine and Jilin University College of Pharmacy.

\section{Treatment}

Abdominal rubbing, massaging, and acupoint pressing (RN6 Qi Hai, RN4 Guan Yuan, RN12 Zhongwan, and ST25 Tianshu) of rats in AM group were performed for 15 min, once a day, for 4 weeks [28]. Information on the positioning of acupoints was obtained from "The appendix of Experimental Acupuncture." Before conduction of the treatment, operators were subjected to training using type TPA-II massage measurement instrument (purchased from Yilian Medical Development Co., Ltd, Shanghai) to learn the correct massage technique and application of pressure, (instrument measuring $0.5 \mathrm{~kg}$ ). The RSV group rats were administered with $260 \mathrm{mg} / \mathrm{kg}$ RSV orally once a day, for 4 weeks. Control groups were orally administered with an equal dose of normal saline once a day, for 4 weeks. 
The weight of the rats and food intake were measured weekly. After the treatment, all rats were anesthetized to obtain blood samples and were sacrificed thereafter. The quadriceps femoris from all rats was removed and stored in liquid nitrogen for further analysis.

\section{Analyses of Biochemical Indicators}

Fasting blood glucose (FBG) levels were measured using the glucose oxidase method (AICARE, China). Serum levels of TC, TG, HDL-C, and LDL-C were measured using a biochemical marker kit. The levels of fasting serum insulin (FINS), FFA, ADPN, TNF- $\alpha$, and IL- 6 were determined by performing ELISA. The homeostasis model assessment of insulin resistance (HOMA-IR) was calculated using the formula HOMA-IR $=$ FBG $\times$ FINS/22.5.

\section{qPCR Analysis}

Total RNA from the samples was isolated using an RNA extraction kit according to the manufacturer's instructions. RNA reverse transcription and amplification were performed using the TransScript ${ }^{\circ}$ Green Two-Step qRT-PCR SuperMix. mRNA expression was detected using Stratagene Mx3000p (Agilent, USA). SIRT1 (forward: 5'-CAG CAT CTT GCC TGA TTT GTA A-3', reverse: 5'-TTG GAA TTA GTG CTA CTG GTC TTA C- $3^{\prime}$ ) and PGC- $1 \alpha$ (forward: 5'-CATTCAGGAGCTGGATGGCT- ${ }^{\prime}$, reverse 5'-TATGTTCGCGGGCTCATTGT-3') mRNA expression levels were calculated by using $2^{-\Delta \Delta \mathrm{Ct}}$ and compared with those of $\beta$-actin (forward: $5^{\prime}$-CGT TGA CAT CCG TAA AGA CCT C- ${ }^{\prime}$, reverse: $5^{\prime}$-TAG GAG CCA GGG CAG TAA TAT-3') used as an internal control.

\section{Western Blotting}

Briefly, total protein was extracted using the RIPA lysis buffer, and protein concentration was detected by performing the BCA method. Protein $(30 \mathrm{mg})$ was electroblotted onto a polyvinylidene fluoride (PVDF) membrane following separation on $8 \%$ SDS-polyacrylamide gel. The PVDF membrane was incubated with blocking solution $(5 \%$ skim milk) for $1 \mathrm{~h}$ and overnight with antibodies $(1: 500)$ at $4{ }^{\circ} \mathrm{C}$. Blots were subjected to washing steps using Tris buffer solution (TBS) and incubated with HRP-conjugated secondary antibody (1:5000). Washing steps were subsequently performed using TBS and blots were developed using the electrochemiluminescence method; results were determined using a chemiluminescence imaging system (Bio-Rad, 1708370). The ImageJ software was used for analysis and semi-quantification of the blots.

\section{Statistical Analysis}

Data are presented as mean \pm standard deviation (SD). Statistical analyses were performed using SPSS 22.0 and GraphPad Prism 8. Comparison between groups was performed using unpaired two-tailed Student's $t$ test and ANOVA. A $p$ value less than 0.05 was considered as statistically significant.

\section{Results}

\section{AM Alleviates IR in HFD-Fed Rats}

The growth parameters measured in rats are shown in Table 1 and Fig. 1. Symptom severity was assessed by using the Lee index and HOMA-IR. HFD led to a significant increase in the rat body weight. Among the HFD groups, the Lee index was significantly lower in the RSV and AM groups than the model group $(p<0.05)$. HFD group rats showed significantly greater HOMA-IR after 4 weeks of AM intervention $(p<0.05)$. Although not statistically significant, AM resulted in a weight loss in the AM group rats.
Table 1 Am ameliorates the state of HFD-induced IR rats

\begin{tabular}{lcccc}
\hline & CON & RSV & MOD & AM \\
\hline Weight $(\mathrm{g})$ & $404.8 \pm 39.21$ & $413.8 \pm 96.07^{*}$ & $498.70 \pm 66.45^{\Delta}$ & $446.50 \pm 33.30$ \\
Length $(\mathrm{cm})$ & $24.92 \pm 1.03$ & $23.83 \pm 3.34$ & $24.38 \pm 0.87$ & $24.67 \pm 0.87$ \\
Lee index & $2.84 \pm 0.10$ & $2.97 \pm 0.22^{*}$ & $3.24 \pm 0.19^{\Delta}$ & $3.11 \pm 0.11$ \\
FBG $(\mathrm{mmol} / \mathrm{L})$ & $6.28 \pm 0.58$ & $12.03 \pm 6.26^{*}$ & $14.63 \pm 7.9^{\Delta}$ & $13.93 \pm 5.86^{*}$ \\
FINS $(\mathrm{mU} / \mathrm{L})$ & $6.90 \pm 1.08$ & $7.59 \pm 0.93^{\Delta}$ & $10.08 \pm 2.65^{\Delta}$ & $8.90 \pm 0.72^{*}$ \\
HOMA-IR & $1.98 \pm 0.39$ & $5.19 \pm 2.64^{*}$ & $9.62 \pm 2.78^{\Delta}$ & $5.47 \pm 2.42^{*}$ \\
\hline
\end{tabular}

All resulting data were statistically analyzed using a two-tailed Student's $t$ test. Data are expressed as means $\pm \mathrm{SD}(n=12 /$ groups $)$

CON control, RSV resveratrol, AM abdominal massage, FINS fasting insulin, HOMA-IR homeostasis model assessment-insulin resistance

$* P<0.05$ was presented a significant difference with MOD group; ${ }^{\Delta} P<0.05$ was presented a significant difference with CON group 
Fig. 1 AM alleviates IR in HFDfed rats. A Weight, B length, C Lee Index, D FBG, E FINS, and F HOMA-IR indices. Data are expressed as means $\pm \mathrm{SD}$. Different superscript letters indicate statistically significant differences between the groups $(P<0.05)$. $* P<0.05$ represented a significant difference with MOD group; ${ }^{\Delta} P<0.05$ represented a significant difference with CON group. CON control, RSV resveratrol, AM abdominal massage
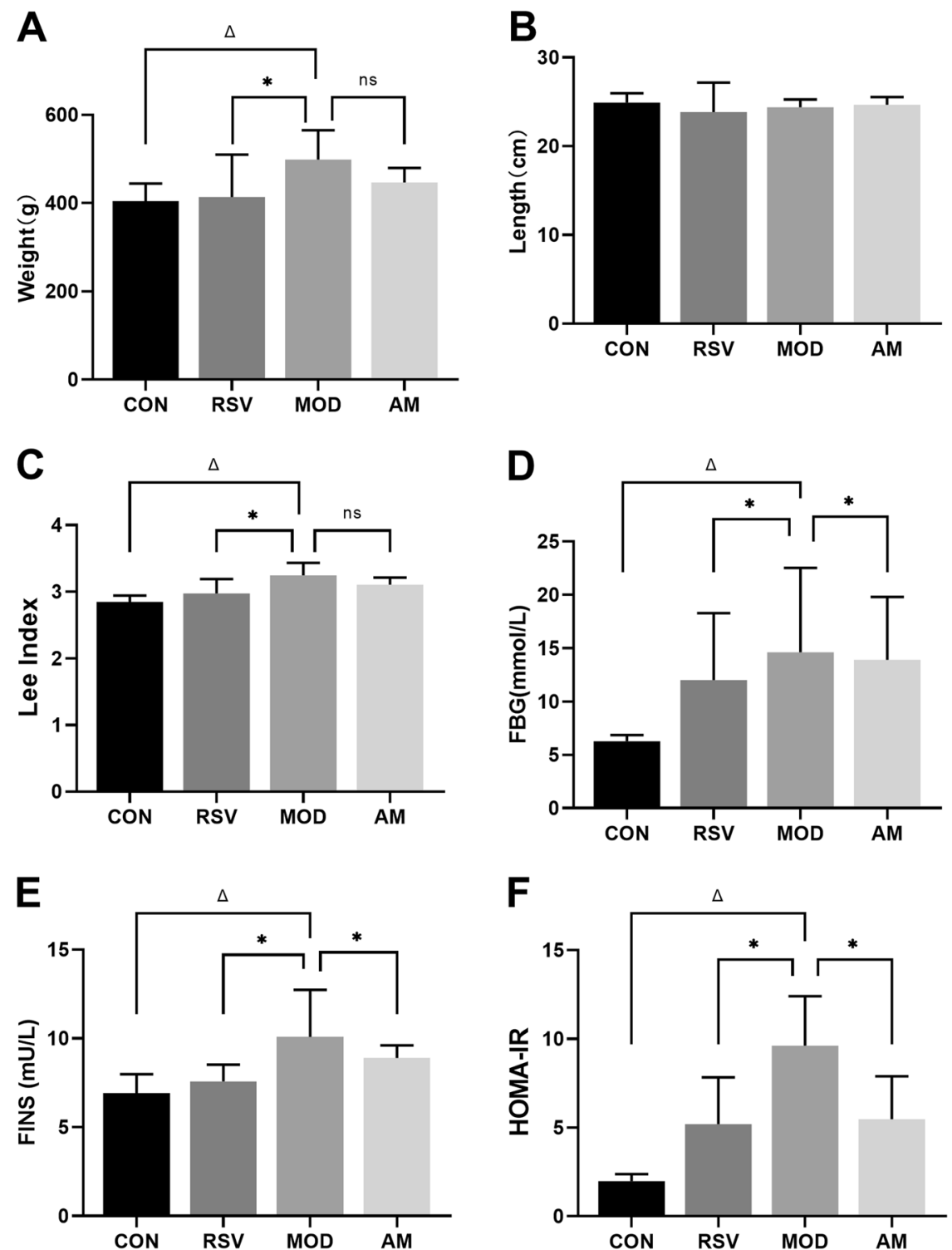

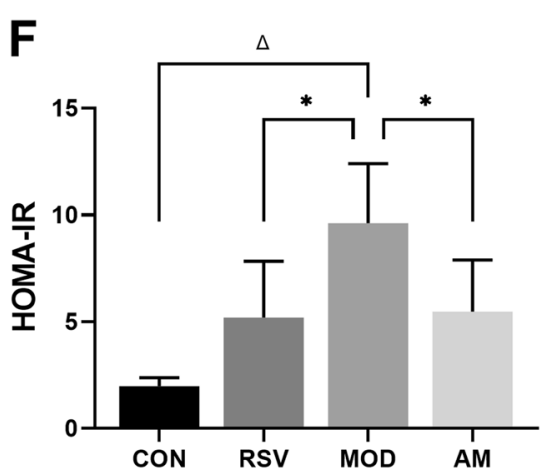

Table 2 Effect of AM on serum lipid accumulation of the HFDinduced obese rats

\begin{tabular}{lllll}
\hline & CON & RSV & MOD & AM \\
\hline TG $(\mathrm{mmol} / \mathrm{L})$ & $0.54 \pm 0.12$ & $0.61 \pm 0.10^{*}$ & $1.25 \pm 0.24^{\Delta}$ & $0.81 \pm 0.20^{*}$ \\
TC $(\mathrm{mmol} / \mathrm{L})$ & $2.04 \pm 0.49$ & $2.68 \pm 0.31^{*}$ & $3.87 \pm 0.19^{\Delta}$ & $3.09 \pm 0.31^{*}$ \\
LDL-C $(\mathrm{mmol} / \mathrm{L})$ & $1.16 \pm 0.08$ & $1.28 \pm 0.10^{*}$ & $1.60 \pm 0.09^{\Delta}$ & $1.35 \pm 0.08^{*}$ \\
HDL-C $(\mathrm{mmol} / \mathrm{L})$ & $0.83 \pm 0.09$ & $0.65 \pm 0.09^{*}$ & $0.28 \pm 0.07^{\Delta}$ & $0.59 \pm 0.06^{*}$ \\
\hline
\end{tabular}

The data represent the means $\pm \mathrm{SD}(n=12$ /groups $)$ of at least three independent experiments. All resulting data were statistically analyzed using a two-tailed Student's $t$ test

CON control, RSV resveratrol, $A M$ abdominal massage, TC total cholesterol, $T G$ triglycerides, $H D L-C$ high-density lipoprotein-cholesterol, $L D L-C$ low-density lipoprotein-cholesterol

${ }^{*} P<0.05$ was presented a significant difference with MOD group; ${ }^{\Delta} P<0.05$ was presented a significant difference with CON group

\section{Effect of AM on Serum Lipid Accumulation in HFD- Induced IR Rats}

As shown in Table 2 and Fig. 2, after 12 weeks HFD feeding, serum TG, TC, and LDL-C levels were found to be significantly higher in the model group as compared to those in the control group rats $(P<0.05)$. In addition, HDL-C levels were found to be lower in the model group rats. Moreover, with the intervention of AM and RSV, the degree of serum lipid accumulation in AM and RSV groups was significantly lower than that in the model group $(P<0.05)$. Our results confirmed that the consumption of a long-term HFD diet leads to the development of dyslipidemia in rats, and continuous AM can effectively alleviate this symptom. 
Fig. 2 Effect of AM on serum lipid accumulation in HFDinduced IR rats. A TG, B TC, C LDL-C, and D HDL-C. Data are expressed as means $\pm \mathrm{SD}$. Different superscript letters indicate statistically significant differences between the groups $(P<0.05) . * P<0.05$ represented a significant difference with MOD group; ${ }^{\Delta} P<0.05$ represented a significant difference with CON group. CON control, RSV resveratrol, AM abdominal massage
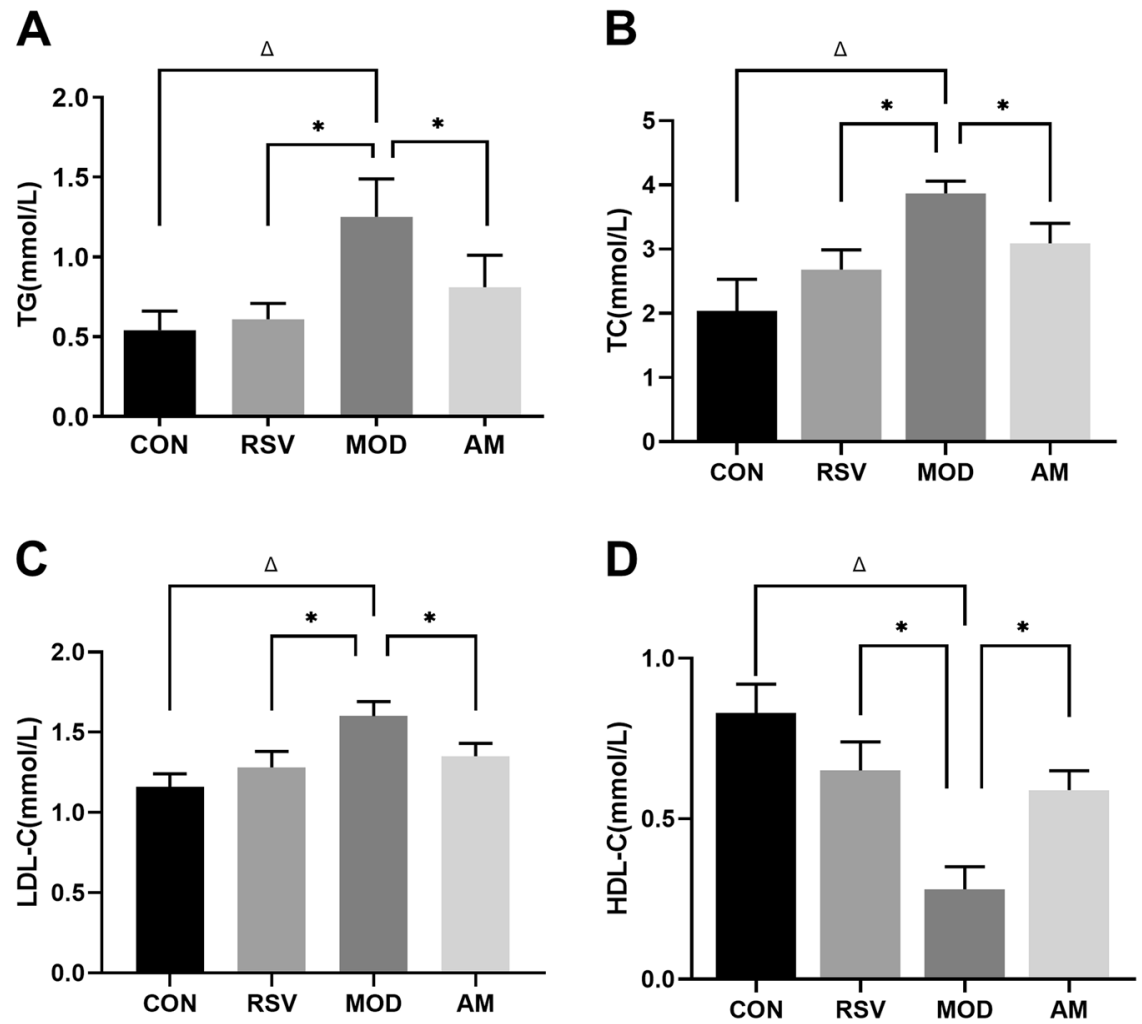

Table 3 AM maintains a balance of the secretion of proinflammatory cytokines and adipocytokines in HFD-induced IR rats

\begin{tabular}{ccccc}
\hline & CON & RSV & MOD & AM \\
\hline ADPN $(\mu \mathrm{g} / \mathrm{mL})$ & $14.73 \pm 3.10$ & $15.25 \pm 2.19^{*}$ & $12.24 \pm 1.53^{\Delta}$ & $14.14 \pm 1.84^{*}$ \\
FFA $(\mu \mathrm{mol} / \mathrm{L})$ & $438.30 \pm 75.41$ & $399.45 \pm 51.22^{*}$ & $502.86 \pm 28.26^{\Delta}$ & $418.89 \pm 61.64^{*}$ \\
TNF- $\alpha(\mathrm{pg} / \mathrm{mL})$ & $206.57 \pm 13.23$ & $225.67 \pm 7.29^{*}$ & $255.27 \pm 5.91^{\Delta}$ & $230.52 \pm 11.94^{*}$ \\
IL-6 $(\mathrm{pg} / \mathrm{mL})$ & $105.70 \pm 7.37$ & $125.13 \pm 9.97^{*}$ & $150.51 \pm 22.64^{\Delta}$ & $133.98 \pm 6.89^{*}$ \\
\hline
\end{tabular}

The data represent the means $\pm \mathrm{SD}$ ( $n=12$ /groups) of at least three independent experiments. All resulting data were statistically analyzed using a two-tailed Student's $t$ test.

$C O N$ control, $R S V$ resveratrol, $A M$ abdominal massage, FFA free fatty acids, $A D P N$ adiponectin, $T N F-\alpha$ tumor necrosis factor- $\alpha, I L-6$ Interleukin- 6

$* P<0.05$ was presented a significant difference with MOD group; ${ }^{\Delta} P<0.05$ was presented a significant difference with CON group

\section{AM Maintains A Balance of Pro-Inflammatory Cytokine Secretion and Adipocytokines in HFD- Induced IR in SD Rats}

We analyzed the levels of pro-inflammatory cytokines and adipocytokines, such as TNF- $\alpha$, IL-6, FFA, and ADPN, in the serum of the rats by performing ELISA. As shown in Table 3 and Fig. 3, the model group had significantly higher serum levels of TNF- $\alpha$, IL- 6 , and FFA as compared to the control group $(P<0.05)$, while ADPN was significantly lower $(P<0.05)$ in the model group. After subjection to 4 weeks of AM or RSV treatment, a significant decrease in the serum levels of TNF- $\alpha$, IL- 6 , and FFA was observed in $\mathrm{AM}$ and RSV group rats as compared to the model group rats $(P<0.05)$. Furthermore, ADPN was found to be significantly higher in the AM and RSV group rats. The difference in the serum levels of FFA and ADPN is also a hallmark of chronic inflammation. Our results showed that HFD-induced IR could stimulate the secretion of proinflammatory cytokines and adipocytokines in metabolic disorders. AM reduced the levels of pro-inflammatory cytokines in the serum of obese rats and maintained a balance of pro-inflammatory cytokines and adipocytokines in HFD-induced IR.

\section{AM activates the AMPK/SIRT1/PGC-1a signaling pathway in skeletal muscle of HFD-induced IR rats}

The AMPK/SIRT1/PGC-1 $\alpha$ pathway is an important signaling pathway that mediates the pathophysiological process 
Fig. 3 AM maintains a balance of pro-inflammatory cytokine secretion and adipocytokines in HFD-induced IR in SD rats. A FFA, B ADPN, C IL-6, and D TNF- $\alpha$. Data are expressed as means \pm SD. Different superscript letters indicate statistically significant differences between the groups $(P<0.05)$. $* P<0.05$ represented a significant difference with MOD group; ${ }^{\Delta} P<0.05$ represented a significant difference with CON group. CON control, RSV resveratrol, AM abdominal massage
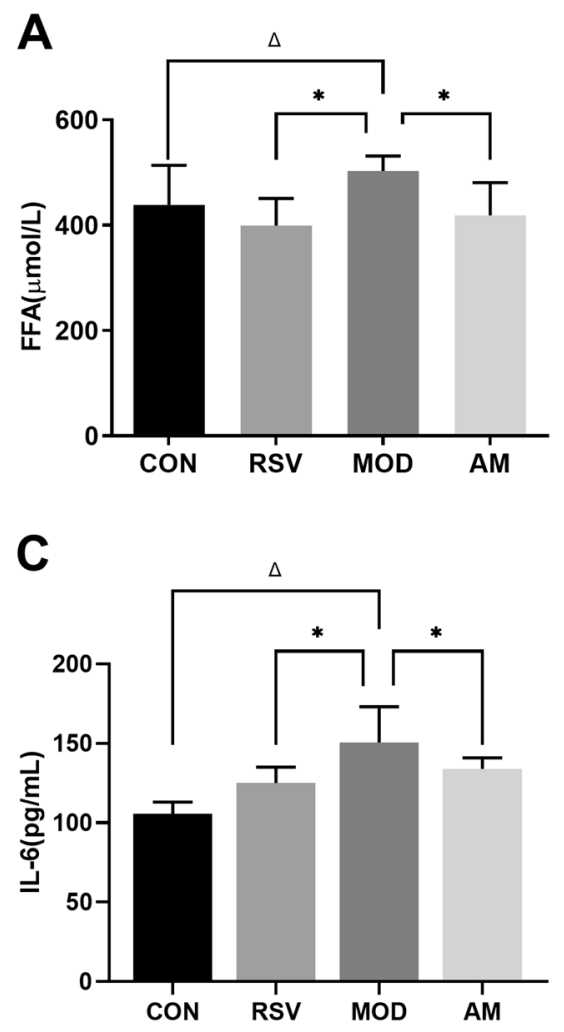
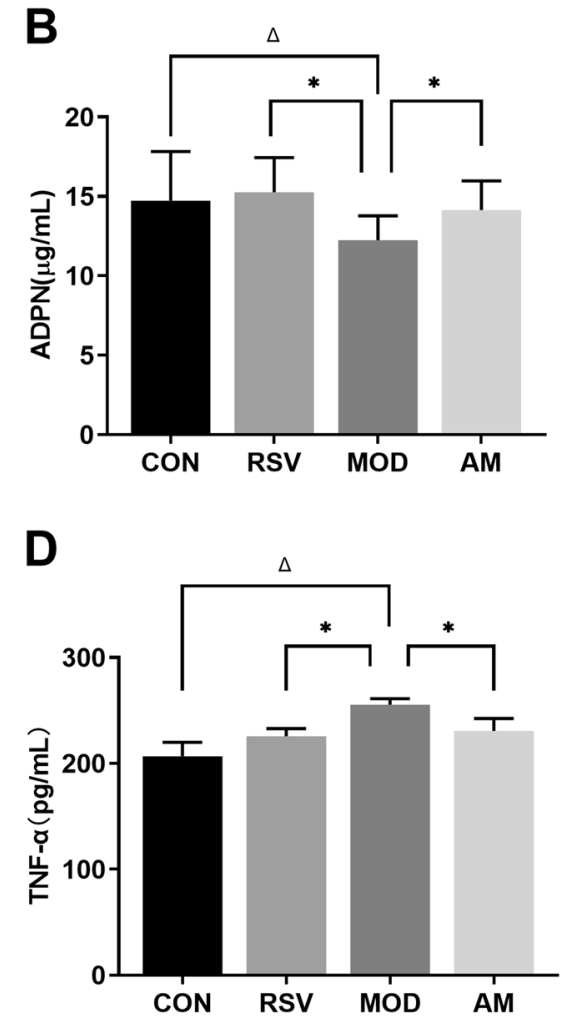

of IR in obesity. In our study, we examined the effect of AM on AMPK regulation in skeletal muscles obtained from HFDinduced obese rats. As depicted in Fig. 4A, HFD feeding in rats markedly decreased p-AMPK, SIRT1, and PGC- $1 \alpha$ protein and mRNA levels in the skeletal muscle of model group rats $(P<0.05)$. However, persistent AM or $\mathrm{RSV}$ treatment reversed this phenomenon by upregulating AMPK phosphorylation levels and by subsequently restoring SIRT1 and PGC- $1 \alpha$ protein and mRNA levels.

\section{Discussion}

In the present study, we investigated the effects of AM on HFD-induced IR.

To the best of our knowledge, this is the first study to evaluate the effect of AM on IR. We aimed to investigate the role of AM in mitigating disordered lipid metabolism in the skeletal muscle of obese rats. Our results showed that both AM and RSV alleviated HFD-induced IR. These results were consistent with those reported by previous studies [30, 31]. It has been reported that free fatty acids with endocrine and local effects (paracrine and autocrine) can affect insulin sensitivity. For example, several factors, such as TNF- $\alpha$, IL-6, leptin, ADPN, IL-1 $\beta$, resistin, retinol-binding protein, and visfatin, are closely related to IR occurrence [32]. In this study, we examined the levels of adipocytokines and pro-inflammatory cytokines in rat serum by performing ELISA. We aimed to study the effect of AM on lipid metabolism and inflammation in the serum of HFD-induced IR in rats. Our results showed that the serum levels of TC, TG, LDL-C, HDL-C, FFA, ADPN, IL-6, and TNF- $\alpha$ were abnormal in model group rats. All the above-mentioned changes indicated that HFD-induced IR rats developed lipid metabolism disorder and inflammation. However, the related adipocytokines and pro-inflammatory cytokines were shown to be positively regulated in the rats treated by AM. Our results indicate that AM can improve the lipid metabolism disorder and IRinduced inflammation in obese rats.

In addition, chronic inflammation caused by obesity is an important cause of IR [33]. In the case of overnutrition, body stores excess energy in the form of fat with the formation of TG, or with an ectopic deposition in the muscle, liver, placenta, and other tissues, thus leading to obesity [34]. Skeletal muscle is one of the most important peripheral insulin target sites [35]. As reported in several studies, TG deposition in skeletal muscle is one of the pivotal markers of IR in body [36, 37]. Thus, when IR occurs, abnormal glucose and lipid metabolism in skeletal muscle are common. AMPK reduces lipid ectopic accumulation by decreasing circulating glucose and fatty acid levels, thus increasing insulin sensitivity [38, 39]. AMPK not only regulates fat generation, but also reduces FFA concentration in the blood, which has an ameliorating effect on lipid abnormalities in obesity [40, 41]. There is an increasing evidence that SIRT1 regulates glucose and lipid metabolism 
Fig. 4 AM activates the AMPK/ SIRT1/PGC-1 $\alpha$ signaling pathway in skeletal muscle of HFD-induced IR rats. A The protein expression of AMPK, p-AMPK, SIRT1, and PGC- $1 \alpha$ in skeleton muscle of IR rats.

B The mRNA expression of SIRT1 and PGC-1 $\alpha$ in skeleton muscle of IR rats. Data are expressed as means $\pm \mathrm{SD}$. Different superscript letters indicate statistically significant differences between the groups $(P<0.05) . * P<0.05$ represented a significant difference with MOD group; ${ }^{\Delta} P<0.05$ represented a significant difference with CON group. CON control, RSV resveratrol, AM abdominal massage
A
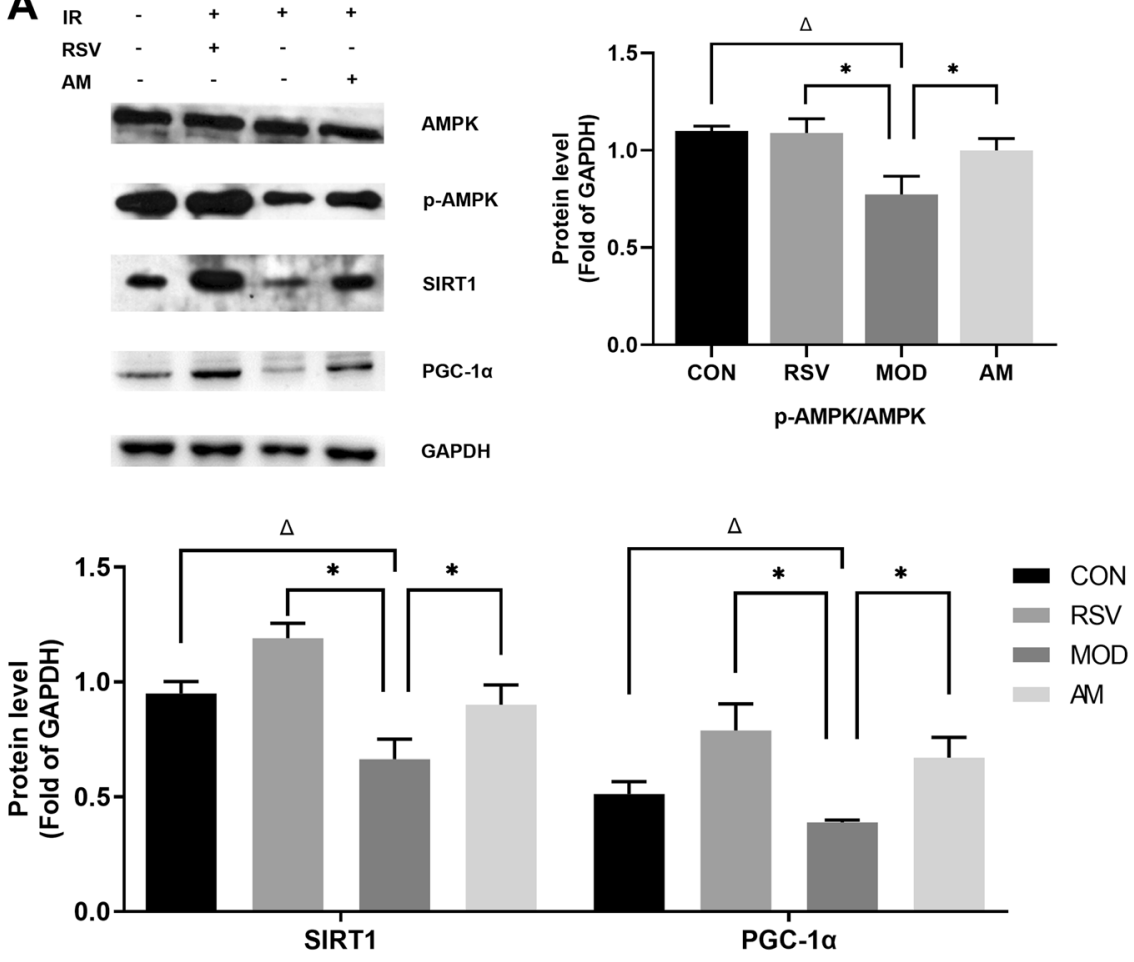

B

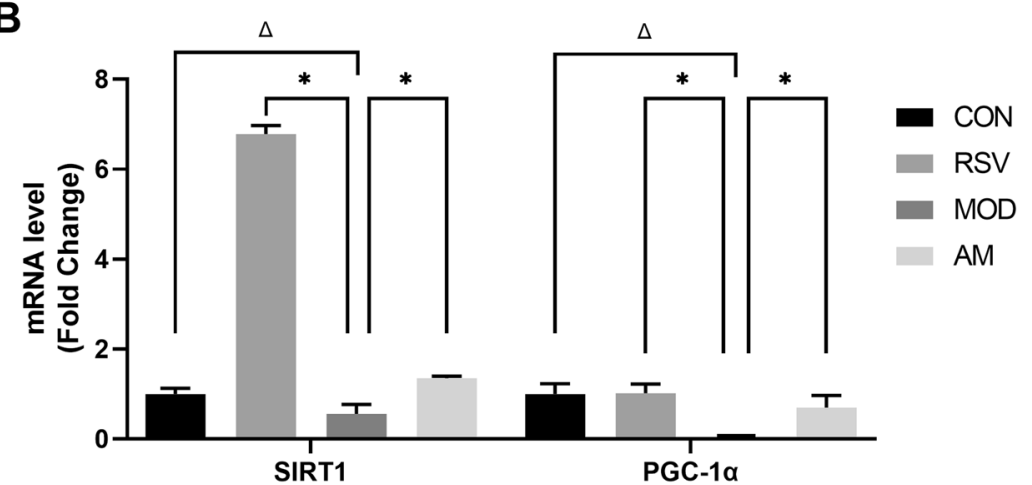

through its deacetylase activity [42]. As reported in several studies, activated SIRT1 improves insulin sensitivity in the liver, skeletal muscle, and adipose tissue, and confers protection to pancreatic beta-cell function and quality[43, 44]. SIRT1 not only plays an important role in cell proliferation, differentiation, senescence, and apoptosis, but also demonstrates a role in regulating glucose and lipid metabolism, inflammatory response, oxidative stress, tumor formation, and cellular metabolic regulation under different conditions of nutritional stress [45]. AMPK and SIRT1 regulate PGC- $1 \alpha$ activity through phosphorylation and deacetylation. Many biological processes, such as mitochondrial biology and fatty acid oxidation, are regulated by the AMPK/SIRT1/PGC-1 $\alpha$ signaling pathway. It also promotes fat oxidation and provides energy for the maintenance of lasting muscle contraction and energy supply and is thus closely associated with the development of IR [46].
Our results showed that $\mathrm{AM}$ increased the expression of p-AMPK, SIRT1, and PGC-1 $\alpha$ in the skeletal muscle of rats, and this indicated that AM activated the AMPK/ SIRT1/PGC- $1 \alpha$ signaling pathway.

As a representative of external therapy commonly used in traditional Chinese medicine, massage therapy can treat patients' endocrine disorders, enhance patients' resting metabolic rate, increase energy consumption, and promote the oxidative utilization of stored fat in the body [47, 48]. It can also enhance blood circulation of the adipose tissue, especially subcutaneous fat, and reduce accumulation. It can also reduce appetite and promote gastrointestinal motility $[17,49]$. Currently, there are no specific clinical drugs or treatment methods available for treating obesity and other metabolic disorders. Therefore, there is an urgent need to develop a safe, effective, eco-friendly, and simple clinical therapy for the treatment of obesity. In recent years, the 
progress witnessed in the treatment of IR in patients with obesity indicates that comprehensive traditional Chinese medicine is gaining prominence as a new therapeutic strategy with increased research focus [50]. Our previous studies [28, 51, 52] have found that AM can significantly improve weight, body mass index, and other indicators of obesity in animal models and obese patients. In this study, our results showed that continuous AM could decrease the secretion of lipids and pro-inflammatory cytokines in the serum of IR rats and could reduce inflammation.

\section{Conclusion}

Taken together, our study proved that AM can help regulate lipid metabolism and reduce inflammation. Our findings suggested that the regulation of IR by AM might be related to the regulation of AMPK/SIRT1/PGC- $1 \alpha$-mediated lipid metabolism and pro-inflammatory factor secretion. These findings provide novel insights into the development of AM as a potential therapy for the improvement of IR-related metabolic disorders, such as T2DM. However, the exact mechanism by which AM results in the treatment of IRassociated complications in other peripheral tissues remains to be elucidated. To this end, further refined studies are warranted to determine its potential for clinical application in obesity treatment.

\section{Compliance with Ethical Standards}

Conflict of Interest The authors declare no competing interests.

Publisher's note Springer Nature remains neutral with regard to jurisdictional claims in published maps and institutional affiliations.

Open Access This article is licensed under a Creative Commons Attribution 4.0 International License, which permits use, sharing, adaptation, distribution and reproduction in any medium or format, as long as you give appropriate credit to the original author(s) and the source, provide a link to the Creative Commons license, and indicate if changes were made. The images or other third party material in this article are included in the article's Creative Commons license, unless indicated otherwise in a credit line to the material. If material is not included in the article's Creative Commons license and your intended use is not permitted by statutory regulation or exceeds the permitted use, you will need to obtain permission directly from the copyright holder. To view a copy of this license, visit http://creativecommons. org/licenses/by/4.0/.

\section{References}

1. Barazzoni, R., Gortan Cappellari, G., Ragni, M., \& Nisoli, E. (2018). Insulin resistance in obesity: an overview of fundamental alterations. Eating and Weight Disorders, 2018(2), 149-157.

2. Abu Bakar, M. H., Shariff, K. A., Tan, J. S., \& Lee, L. K. (2020). Celastrol attenuates inflammatory responses in adipose tissues and improves skeletal muscle mitochondrial functions in high fat dietinduced obese rats via upregulation of AMPK/SIRT1 signaling pathways. European Journal of Pharmacology, 2020, 173371.

3. Banerjee, J., Bruckbauer, A., \& Zemel, M. B. (2016). Activation of the AMPK/Sirt1 pathway by a leucine-metformin combination increases insulin sensitivity in skeletal muscle, and stimulates glucose and lipid metabolism and increases life span in Caenorhabditis elegans. Metabolism, 2016(11), 1679-1691.

4. Sun, Y., Li, J., Xiao, N., Wang, M., Kou, J., \& Qi, L., et al. (2014). Pharmacological activation of AMPK ameliorates perivascular adipose/endothelial dysfunction in a manner interdependent on AMPK and SIRT1. Pharmacological Research, 2014, 19-28.

5. Shiota, A., Shimabukuro, M., Fukuda, D., Soeki, T., Sato, H., \& Uematsu, E., et al. (2012). Telmisartan ameliorates insulin sensitivity by activating the AMPK/SIRT1 pathway in skeletal muscle of obese db/db mice. Cardiovascular Diabetology, 2012, 139.

6. Iwabu, M., Yamauchi, T., Okada-Iwabu, M., Sato, K., Nakagawa, T., \& Funata, M., et al. (2010). Adiponectin and AdipoR1 regulate PGC-1alpha and mitochondria by $\mathrm{Ca}(2+)$ and AMPK/SIRT1. Nature, 2010(7293), 1313-1319.

7. Hörbelt, T., Knebel, B., Fahlbusch, P., Barbosa, D., de Wiza, D. H., \& Van de Velde, F., et al. (2019). The adipokine sFRP4 induces insulin resistance and lipogenesis in the liver. Biochimica et Biophysica Acta - Molecular Basis of Disease, 2019(10), 2671-2684.

8. Trojnar, M., Patro-Małysza, J., Kimber-Trojnar, Ż., LeszczyńskaGorzelak, B., \& Mosiewicz, J. (2019). Associations between fatty acid-binding protein 4 - a proinflammatory adipokine and insulin resistance, gestational and type 2 diabetes mellitus. Cells, 2019, 3 .

9. Rosen, E. D., \& Spiegelman, B. M. (2006). Adipocytes as regulators of energy balance and glucose homeostasis. Nature, 2006 (7121), 847-853.

10. Tilg, H., \& Moschen, A. R. (2006). Adipocytokines: mediators linking adipose tissue, inflammation and immunity. Nature Reviews Immunology, 2006(10), 772-783.

11. Fock, K. M., \& Khoo, J. (2013). Diet and exercise in management of obesity and overweight. Journal of Gastroenterology \& Hepatology, 2013, 59-63.

12. Gao, X., Sun, G., Randell, E., Tian, Y., \& Zhou, H. (2020). Systematic investigation of the relationships of trimethylamine Noxide and L-carnitine with obesity in both humans and rodents. Food \& Function, 2020(9), 7707-7716.

13. Field, T. (2016). Massage therapy research review. Complementary Therapies in Clinical Practice, 2016, 19-31.

14. Field, T. (2014). Massage therapy research review. Complementary Therapies in Clinical Practice, 2014(4), 224-229.

15. Ali, A., Rosenberger, L., Weiss, T. R., Milak, C., \& Perlman, A. I. (2017). Massage therapy and quality of life in osteoarthritis of the knee: a qualitative study. Pain Medicine, 2017(6), 1168-1175.

16. Lu, W. P., Tsai, W. H., Lin, L. Y., Hong, R. B., \& Hwang, Y. S. (2019). The beneficial effects of massage on motor development and sensory processing in young children with developmental delay: a randomized control trial study. Developmental Neurorehabilitation, 2019(7), 487-495.

17. Donoyama, N., Suoh, S., \& Ohkoshi, N. (2018). Adiponectin increase in mildly obese women after massage treatment. Journal of Alternative and Complementary Medicine, 2018(7), 741-742.

18. Kjl, A., Jip, B., \& Syo, A. (2021). The effects of extracorporeal shock wave therapy vs hand massage on serum lipids in overweight and obese women. Annals of Medicine and Surgery (Lond), 63, 102057

19. Di, R., Jingjing, L., \& Junchang, L. et al. (2021). Acupoint massage can effectively promote the recovery of gastrointestinal function after gynecologic laparoscopy. Journal of Investigative Surgery, 34(1), 91-95. 
20. Sut, N., \& Kahyaoglu-Sut, H. (2017). Effect of aromatherapy massage on pain in primary dysmenorrhea: a meta-analysis. Complementary Therapies in Clinical Practice, 2017, 5-10.

21. Avetisyan, M., Schill, E. M., \& Heuckeroth, R. O. (2015). Building a second brain in the bowel. Journal of Clinical Investigation, 2015(3), 899-907.

22. Un, Hyo, \& Baek (2015). The effects of aerobic exercise and abdominal massage on the blood vessel inflammation markers of the middle obesity women. The Korean Journal of Growth and Development, 2015(2), 129-134.

23. Han, G.-S., Kim, G.-D., \& Kang, M.-H. (2014). Effects of myofascial release massage therapy on the changes in blood lipids, blood pressure, and intra-abdominal fat in middle-aged obese women metabolic syndrome risk factors. Korean Journal of Sports Science, 2014(6), 1395-1406.

24. Montejo, G., González-Quirarte, G., Vázquez, C., Sánchez, D., \& Quirarte, N. (2016). Acupuntura y masaje: auxiliar en el tratamiento de pacientes con hipertension y diabetes en el Ejido Cuitláhuac de Tacotalpa. Revista Enfermería Actual En Costa Rica, 2016, 31.

25. Li, H., Wang, J. G., Zhang, W., Zhao, N., Hai, X. H., Sun, S. Q., Sun, Q., Han, Y. H., Zhang, R. C., \& Ma, F. (2017). Chronic fatigue syndrome treated by the traditional Chinese procedure abdominal tuina: a randomized controlled clinical trial. Journal of Traditional Chinese Medicine, 2017(6), 103-110.

26. Moraska, A. F., Hickner, R. C., Rzasa-Lynn, R., Shah, J. P., Hebert, J. R., \& Kohrt, W. M. (2018). Increase in lactate without change in nutritive blood flow or glucose at active trigger points following massage: a randomized clinical trial. Archives of Physical Medicine and Rehabilitation, 2018(11), 2151-2159.

27. Lyu, W. B., Gao, Y., Cheng, K. Y., Wu, R., \& Zhou, W. Q. (2019). Effect of self-acupoint massage on blood glucose level and quality of life in older adults with type 2 diabetes mellitus: a randomized controlled trial. Journal of Gerontological Nursing, 2019(8), 43-48.

28. Han, Y., Yan, M., Liu, H., \& Liu, M. (2020). Effect of abdominal transport and meridian massage on regulating insulin resistance in rats through SIRT1. China Journal of Traditional Chinese Medicine and Pharmacy, 2020(05), 2568-2571. in Chinese.

29. Prada, P. O., Zecchin, H. G., Gasparetti, A. L., Torsoni, M. A., Ueno, M., \& Hirata, A. E., et al. (2005). Western diet modulates insulin signaling, c-Jun N-terminal kinase activity, and insulin receptor substrate-1ser307 phosphorylation in a tissue-specific fashion. Endocrinology, 2005(3), 1576-1587.

30. Jimenez-Gomez, Y., Mattison, J. A., Pearson, K. J., MartinMontalvo, A., Palacios, H. H., \& Sossong, A. M., et al. (2013). Resveratrol improves adipose insulin signaling and reduces the inflammatory response in adipose tissue of rhesus monkeys on high-fat, high-sugar diet. Cell Metabolism, 2013(4), 533-545.

31. Wändell, P. E., Ärnlöv, J., Nixon Andreasson, A., Andersson, K., Törnkvist, L., \& Carlsson, A. C. (2013). Effects of tactile massage on metabolic biomarkers in patients with type 2 diabetes. Diabetes \& Metabolism, 2013(5), 411-417.

32. Kralisch, S., Klein, J., Bluher, M., Paschke, R., Stumvoll, M., \& Fasshauer, M. (2005). Therapeutic perspectives of adipocytokines. Expert Opinion on Pharmacotherapy, 2005(6), 863-872.

33. Wellen, K. E., \& Hotamisligil, G. S. (2005). Inflammation, stress, and diabetes. The Journal of clinical investigation, 2005(5), 1111-1119.

34. Olefsky, J. M., \& Glass, C. K. (2010). Macrophages, inflammation, and insulin resistance. Annual Review of Physiology, 2010, 219-246.
35. Liang, F., \& Koya, D. (2010). Acupuncture: is it effective for treatment of insulin resistance? Diabetes, Obesity and Metabolism, 2010(7), 555-569.

36. Capeau, J. (2008). Insulin resistance and steatosis in humans. Diabetes \& Metabolism, 2008(6 Pt 2), 649-657.

37. Frayn, K. N. (1998). Regulation of fatty acid delivery in vivo. Advances in Experimental Medicine and Biology, 1998, 171-179.

38. Kim, M. S., Hur, H. J., Kwon, D. Y., \& Hwang, J.-T. (2012). Tangeretin stimulates glucose uptake via regulation of AMPK signaling pathways in $\mathrm{C} 2 \mathrm{C} 12$ myotubes and improves glucose tolerance in high-fat diet-induced obese mice. Molecular and Cellular Endocrinology, 2012(1), 127-134.

39. Fujii, N., Ho, R. C., Manabe, Y., Jessen, N., Toyoda, T., \& Holland, W. L., et al. (2008). Ablation of AMP-activated protein kinase alpha2 activity exacerbates insulin resistance induced by high-fat feeding of mice. Diabetes, 2008(11), 2958-2966.

40. Crabb, D. W., \& Liangpunsakul, S. (2006). Alcohol and lipid metabolism. Journal of Gastroenterology \& Hepatology, 2006, S56-S60.

41. Botham, K. M., \& Wheeler-Jones, C. P. (2013). Postprandial lipoproteins and the molecular regulation of vascular homeostasis. Progress in Lipid Research, 2013(4), 446-464.

42. Cao, Y., Jiang, X., Ma, H., Wang, Y., Xue, P., \& Liu, Y. (2016). SIRT1 and insulin resistance. Journal of Diabetes and Its Complications, 2016(1), 178-183.

43. Cantó, C., \& Auwerx, J. (2009). PGC-1alpha, SIRT1 and AMPK, an energy sensing network that controls energy expenditure. Current Opinion in Lipidology, 2009, 2.

44. Zhou, S., Tang, X., \& Chen, H. Z. (2018). Sirtuins and insulin resistance. Frontiers in Endocrinology, 2018, 748.

45. Myers, M. J., Shepherd, D. L., Durr, A. J., Stanton, D. S., Mohamed, J. S., \& Hollander, J. M., et al. (2019). The role of SIRT1 in skeletal muscle function and repair of older mice. Journal of Cachexia, Sarcopenia and Muscle, 2019(4), 929-949.

46. Goodarzi, M. O., \& Bryer-Ash, M. (2005). Metformin revisited: re-evaluation of its properties and role in the pharmacopoeia of modern antidiabetic agents. Diabetes, Obesity and Metabolism, 2005(6), 654-665.

47. Field, T., Hernandez-Reif, M., Diego, M., Schanberg, S., \& Kuhn, C. (2005). Cortisol decreases and serotonin and dopamine increase following massage therapy. International Journal of Neuroscience, 2005(10), 1397-1413.

48. Jung, G. S., Choi, I. R., Kang, H. Y., \& Choi, E. Y. (2017). Effects of meridian acupressure massage on body composition, edema, stress, and fatigue in postpartum women. Journal of Alternative and Complementary Medicine, 2017(10), 787-793.

49. Weerapong, P., Hume, P. A., \& Kolt, G. S. (2005). The mechanisms of massage and effects on performance, muscle recovery and injury prevention. Sports Medicine, 2005(3), 235-256.

50. Martinez, B., \& Peplow, P. V. (2016). Treatment of insulin resistance by acupuncture: a review of human and animal studies. Acupuncture in Medicine, 2016(4), 310-319.

51. Chen, S., Zhong, C., Zhang, X., Zhang, X., Zhuo, Y., \& Liu, M. (2020). Optimal application of abdominal transport and meridian in the treatment of simple obesity. Lishizhen Medicine and Materia Medica Research, 2020(01), 142-143. in Chinese.

52. Lyu, W., \& Liu, M. (2019). Study on the optimization of abdominal massage frequency in the treatment of simple obesity. Journal of Changchun University of Chinese Medicine, 2019(03), 479-82. in Chinese. 